\title{
Changes in ECG among Patients with Drug Induced Poisoning in a Tertiary Care Hospital
}

\author{
Rahman $\mathrm{AU}^{1^{*}}$, Chowdhury $\mathrm{AW}^{2}$, Jabeen $\mathrm{S}^{3}$ \\ ${ }^{1}$ Department of Cardiology, National Institute of Cardiovascular Diseases, Dhaka, Bangladesh; \\ ${ }^{2}$ Department of Cardiology, Dhaka Medical College, Dhaka, Bangladesh; ${ }^{3}$ International Centre for \\ Diarrhoeal Disease Research, Dhaka, Bangladesh
}

\begin{abstract}
Background:A wide variety of ECG changes can be seen with cardiac and noncardiac agents and may occur at therapeutic or toxic levels. Wide QRS and QT prolongation may be seen in poisoning cases; are potentially dangerous and indicate the necessity of continuous cardiac monitoring. The objective of this study was to determine the changes in ECG among patients admitted with pharmaceutical drug induced poisoning in a tertiary care hospital in Dhaka.

Methods: This cross sectional study was carried out at the Department of Medicine, Dhaka Medical College Hospital (DMCH) during July-December, 2013. Detailed information were obtained; clinical examination and relevant investigations including ECG were done in each case according to protocol.

Results: Among 66 cases mean age was 22.9 ( \pm 6.47$)$ years and male to female ratio was 1: $2.14(21 \mathrm{Vs} 45)$. Common drug groups taken by the study population were benzodiazepines in $31(47.0 \%)$ and tricyclic antidepressants (TCA) in 18(27.3\%) cases.Normal ECG findings were found in $28(42.4 \%)$ cases, 18 $(27.3 \%)$ cases revealed sinus tachycardia, 11(16.7\%) showed prolong QT Interval, 6(9.1\%) had atrial tachycardia, 5(7.6\%) showed wide QRS and 3(4.6\%) cases revealed prolong PR interval. Patients withTCA poisoning developedsinus tachycardia in 11(61.1\%), prolong QT Interval in 6(33.3\%), wide QRS in $5(27.8 \%)$, atrial tachycardia in 5(27.8\%), prolong PR Interval in 2(11.1\%), and Tall $\mathrm{R}$ in aVRin $2(11.1 \%)$ cases. Prolong QT Interval were found among $3(75.0 \%)$ patients with $\mathrm{K}+$ efflux channel blockers (chlorpromazine, chlorpheniramine and quetiapine) overdose.Patients with beta blocker overdose developed sinus bradycardia in $4(100.0 \%)$ and prolong PR Interval in 1(25.0\%) cases.

Conclusion: Common drugs taken by the patients were amitriptyline and sedatives. Wide QRS, prolong QT interval and some other ECG changes were observed by TCA poisoning in higher doses. TCA, benzodiazepines and K channel blockers induced prolong QT Interval in higher doses.Careful interpretation of ECG findings can provide key information to guide management of the poisoned patients.
\end{abstract}

Keywords: ECG changes, drug induced poisoning, Tertiary care hospital

\section{Introduction}

A wide variety of electrocardiographic (ECG) changes can be seen with cardiac and non-cardiac agents and may occur at therapeutic or toxic levels even in patients without history of cardiac pathology. Most of the drug induced poisoning related mortality is due to cardiac complications like arrhythmia. The diagnosis and management of patients with an abnormal ECG encountered in a specific toxicity can be very challenging even for experienced clinicians. ${ }^{1}$

*Correspondence: Ashraf-Ur-Rahman, Department of Cardiology, National Institute of Cardiovascular Diseases, Dhaka, Bangladesh;

e-mail:drwadud@hotmail.com ORCID: https://orcid.org/0000-0002-9963-0509
The WHO estimates that approximately 3 million poisonings occur annually worldwide and cause more than 220,000 deaths. Developing countries like India and Sri Lanka experiences alarming rates of toxicity and death. ${ }^{2}$ Common poisoning in Bangladesh are organophosphorous compound and street poisoning with ultra-short acting sedative-hypnotics. ${ }^{3}$

Many unrelated drugs share a common cardiac pharmacologic effect if taken in overdose. Some medications involve more than one mechanism of actions and thus may result in a combination 
or myriad of ECG changes. ${ }^{4}$ The main mechanisms involved include membrane depressant action [sodium $\left(\mathrm{Na}^{+}\right)$channel blockers, slow calcium channel blockers, outward potassium $\left(\mathrm{K}^{+}\right)$channel blockers, and $\mathrm{Na}^{+}-\mathrm{K}^{+}$ATPase blockers], and action on autonomic nervous system and its sites of cardiovascular action (beta-adrenergic blockers and other sympathetic-inhibitors, sympathomimetics, anticholinergic and cholinomimetic substances). ${ }^{5}$

In a toxicological situation, QRS complex widening likely results directly from $\mathrm{Na}^{+}$ channel blockage or indirectly from toxininduced hyperkalemia. ${ }^{3} \mathrm{R}$ wave elevation in $\mathrm{aVR} \geq 3 \mathrm{~mm}$ is the only ECG variable that significantly indicates the risk of seizures and arrhythmias in acute tricyclic antidepressant (TCA) poisoning. ${ }^{6}$ In addition, QT interval prolongation can occur with TCA poisoning and $\mathrm{K}$ efflux blocker toxicity. ${ }^{7} \mathrm{QT}$ prolongation is considered to occur when the QTc interval is greater than 0.44 seconds in men and 0.46 seconds in women. ${ }^{8} \mathrm{AV}$ block or an increase in ventricular automaticity are the most common manifestations of Digoxin toxicity and have been shown to occur in $30 \%$ to $40 \%$ of verified cases of toxicity. ${ }^{9}$

The aim of this study was to group together agents that cause similar ECG effects and to find out the different ECG changes, if any, associated with different drugs in overdose. It could help in early detection of cardiac abnormality using a simple diagnostic tool and thereby help us in taking appropriate therapeutic measures in emergency situation. Though there are few papers and review articles regarding ECG changes in drug poisoning in Europe, North America and some parts of Asia; no such study was found in our country.

\section{Materials and Methods}

This cross sectional study was carried out on 66 patients, admitted at the department of medicine, Dhaka Medical College Hospital, due to poisoning from drug overdose. The incidence of drug induced poisoning is unknown in Bangladesh. Patients of both sexes, ages more than 12 years, over a period of July 2013 to
December 2013 were included in the study. Patients with unknown or street poisoning, poisoning due to causes other than drugs, unwilling to participate and known case of IHD, arrhythmia were excluded from the study. Face to face interview was done of patients or their attendants. The diagnosis was based on the definite history of drug ingestion and clinical features. Relevant investigations like RBS, CBC, SGPT, serum bilirubin, prothrombin time, serum creatinine, serum electrolytes were done to exclude other possibilities. 12 lead ECG was carried out immediately on admission of all poisoning patients in the general medicine ward.

ECG analysis included the rate, rhythm, ST/T abnormalities, conduction defects and measurement of P-R, QRS and Q-T intervals. TheQ-T interval was corrected (QTc) according to the formula of Bazett. ${ }^{10}$ Data processing and analyzing were done using appropriate statistical techniques. Descriptive analyses were including done where frequencies and percentages. Ethical clearance was obtained from the Dhaka Medical College authority. Participations of the patients were voluntary and informed written consent was taken.

\section{Results}

Majority of the study population $(86.3 \%)$ were less than 30 years of age (43.9\% within 21-30 yearsand $42.4 \% \leq 20$ years). Females were predominant $(68.25 \%)$ and male female ratio was $1: 2.14$. Majority of the participants were married (57.6\%). 62.1\% patients came from middle class group, while $30.3 \%$ from poor and $7.6 \%$ from higher socio-economic statusrespectively.

Table I: Sex distribution in different drug groups of poisoning $(n=66)$

\begin{tabular}{lccccc}
\hline \multicolumn{1}{c}{ Sex $/$ Drugs $\downarrow$} & \multicolumn{2}{c}{ Male } & \multicolumn{2}{c}{ Females } & Total \\
& $\mathbf{n = 2 5}$ & $\mathbf{\%}$ & $\mathbf{n = 4 5}$ & $\mathbf{\%}$ & $\mathbf{n = 6 6}$ \\
\hline Benzodiazepines & 12 & 57.1 & 19 & 42.2 & 31 \\
Thio benzodiazepines & 0 & 0 & 2 & 4.4 & 2 \\
TCA (inhibitors of Na & 5 & 23.8 & 13 & 28.9 & 18 \\
channels) & 1 & 4.8 & 3 & 6.7 & 4 \\
Beta blockers & 0 & 0 & 1 & 2.2 & 1 \\
H$_{3}$ receptor antagonists & 1 & 4.8 & 2 & 4.4 & 3 \\
NSAIDs & 1 & 4.8 & 3 & 6.7 & 4 \\
K $^{+}$efflux channel & 0 & 0 & 2 & 4.4 & 2 \\
blocker & 1 & 4.8 & 0 & 0 & 1 \\
Anti-histamine & 21 & 100 & 45 & 100 & 66 \\
Na+ -K+ ATPase & & & & & \\
blocker & &
\end{tabular}


Common drug groups taken by the study population were benzodiazepinesin $31(47.0 \%)$ andtricyclic antidepressants (TCA) in $18(27.3 \%)$ patients. Common drugs taken were amitriptyline $\quad 16(24.2 \%)$, bromazepam $11(16.7 \%)$, clonazepam 10(15.2\%) and diazepam $10(15.2 \%)$ respectively.

About half of the patients (42.42\%) had normal ECG. Common ECG changes were sinus tachycardia (28.79\%) and Prolong QT Interval (16.67\%).Some drug groups produced multiple types of ECG changes. Out of 66 study population, 2 patients of TCA poisoning died (table II).

Table II: Number of poisoning cases in each drug $(n=66)$

\begin{tabular}{lccc}
\hline Group of drug & Name & $\begin{array}{c}\text { Number of } \\
\text { poisoning cases }\end{array}$ & \% \\
\hline Benzodiazepines & Bromazepam & 11 & 16.7 \\
& Diazepam & 10 & 15.2 \\
Thio & Clonazepam & 10 & 15.2 \\
benzodiazepines & Olanzepine & 02 & 03.0 \\
TCA (Inhibitors & Amitriptyline & 16 & 24.3 \\
of Na channels) & Nortriptyline & 02 & 3.0 \\
Beta blockers & Atenolol & 03 & 4.5 \\
& Metoprolol & 01 & 1.5 \\
H ${ }_{3}$ receptor & Betahistine & 01 & 01.5 \\
antagonists & Paracetamol & 03 & 04.5 \\
NSAIDs & Chlorpromazine & 01 & 01.5 \\
$\mathrm{~K}^{+}$efflux channel & Chlorpheniramine & 01 & 01.5 \\
blocker & Quetiapine & 01 & 01.5 \\
& Loratadine & 01 & 01.5 \\
Anti-histamine & Cetrizine & 01 & 01.5 \\
Na+ -K+ ATPase & Cyproheptadine & 01 & 01.5 \\
blocker & Digoxin & 01 & 01.5 \\
& Total & 66 & 100.0 \\
\hline
\end{tabular}

Table III:ECG findings of the study population $(n=66)$

\begin{tabular}{lcc}
\multicolumn{1}{c}{ ECG Findings } & Number of cases & $\%$ \\
\hline Normal ECG & 28 & 42.42 \\
Atrial Fibrillation & 01 & 01.52 \\
Atrial Tachycardia & 06 & 09.09 \\
Sinus Tachycardia & 19 & 28.79 \\
Sinus Bradycardia & 04 & 06.06 \\
Wide QRS & 05 & 07.58 \\
Prolong PR Interval & 03 & 04.55 \\
Prolong QT Interval & 11 & 16.67 \\
ST-T depression & 01 & 1.52 \\
T Inversion & 01 & 01.52 \\
Tall R in aVR & 02 & 03.03 \\
\hline
\end{tabular}

Note: some drug groups induced multiple type of ECG changes

Patients with benzodiazepines poisoning revealed mostly normal ECG in 21(67.7\%) and sinus tachycardia in $7(22.6 \%)$ cases. However, about 9.7\% cases revealed abnormal ECG changes which

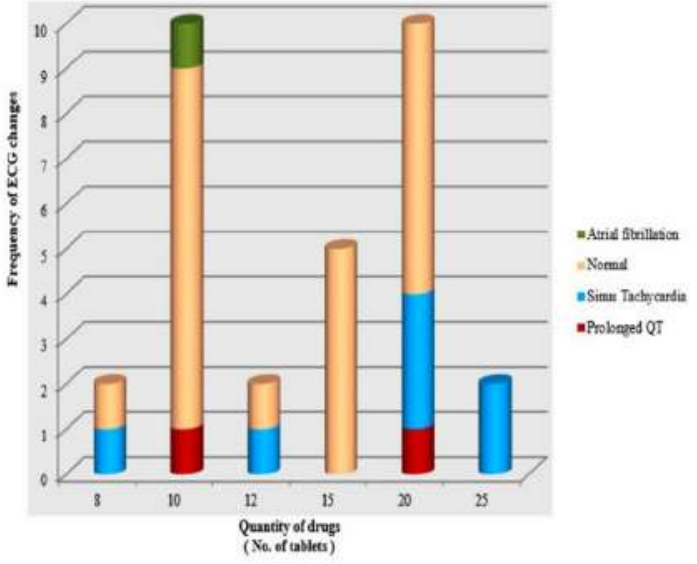

Figure 1: ECG changes in benzodiazepine (bromezepam, clonazepam and diazepam) poisoning cases $(n=31)$

included prolong QT Interval (2 cases, 6.5\%) and atrial fibrillation (1 case, 3.2\%) (figure 1).

ECG findings in TCA poisoning group (figure 2) were sinus tachycardia in 11(61.1\%), prolong QT interval in 6(33.3\%), wide QRS in 5(27.8\%), atrial tachycardia in $5(27.8 \%)$, prolong PR interval in $2(11.1 \%)$, andtall $\mathrm{R}$ in aVR in2(11.1\%) cases. Only 1 patient $(5.5 \%)$ induced normal ECG.

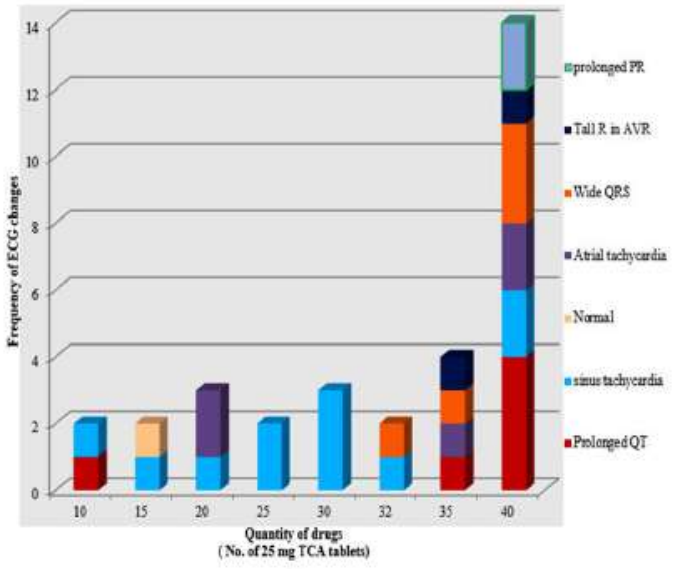

Figure 2: ECG changes in tricyclic antidepressan (amitriptyline and nortriptyline) poisoning cases $(n=18)$

Out of 4 patients of $\mathrm{K}+$ efflux channel blocker poisoning, 1 patient with 10 tablets of Loratadine poisoning were found to have Normal ECG while 20 tablets of Chlorpromazine, Chlorpheniramine and Quetiapine poisoning induced Prolong QT Interval (3 cases, $75.0 \%$ ).

All 4 patients with beta blockersoverdose (figure 3) induced sinus bradycardia. 1 patient $(25.0 \%)$ with 10 tablets of atenolol poisoning was found to reveal prolong PR interval. 


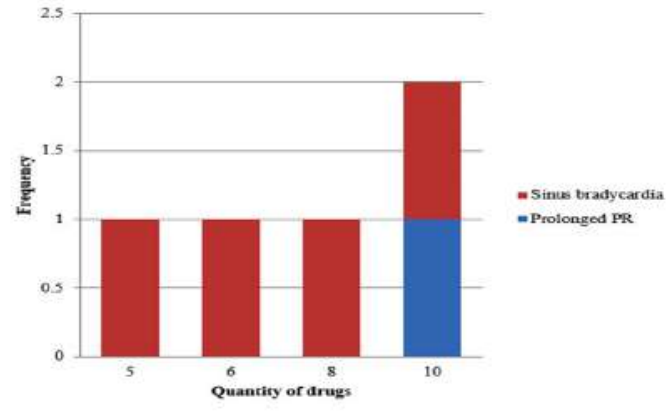

Figure 3: ECG changes in beta blocker poisoning (atenolol 50 mg tablets \& metoprolol $50 \mathrm{mg}$ tablets) cases $(\mathrm{n}=4)$
Anti-histamine $\mathrm{p}$ oisoning cases showed normal ECG findings even with 20 tablets. 10-12 tablets of paracetamol (500 $\mathrm{mg}$ tablets) ingestion revealed ST-T depression and $\mathrm{T}$ inversion in $2 / 3^{\text {rd }}$ cases $(2$ out of 3 cases). One patient with 6 tablets of $\mathrm{Na}+-$ $\mathrm{K}+$ ATPase blocker (digoxin $0.25 \mathrm{mg}$ tablet) overdose developed atrial tachycardia (table IV).

Table IV: Specific ECG changes observed in each group of drugs

\begin{tabular}{|c|c|c|c|c|c|c|c|c|c|c|c|}
\hline \multirow[b]{2}{*}{ ECG findings } & \multirow[b]{2}{*}{ 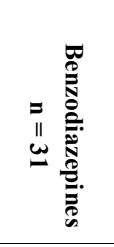 } & \multirow[b]{2}{*}{ 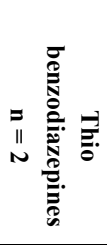 } & \multirow[b]{2}{*}{ 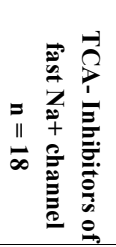 } & \multicolumn{4}{|c|}{ Groups of Drug } & \multirow[b]{2}{*}{ 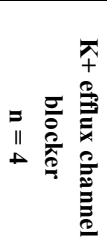 } & \multirow[b]{2}{*}{ 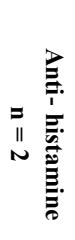 } & \multirow[b]{2}{*}{ 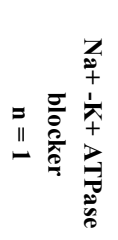 } & \multirow[b]{2}{*}{$\begin{array}{l}=\overrightarrow{0} \\
\stackrel{0}{\partial} \\
\stackrel{2}{0}\end{array}$} \\
\hline & & & & 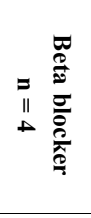 & 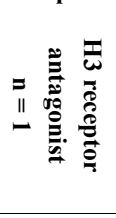 & 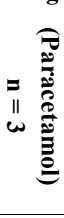 & 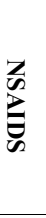 & & & & \\
\hline Atrial Fibrillation & $1(3.23)$ & 0 & 0 & 0 & 0 & 0 & & 0 & 0 & 0 & 1 \\
\hline Atrial Tachycardia & 0 & 0 & $5(27.8)$ & 0 & 0 & 0 & & 0 & 0 & 1 & 6 \\
\hline $\begin{array}{l}\text { Prolong QT } \\
\text { Interval }\end{array}$ & $2(6.45)$ & 0 & $6(33.3)$ & 0 & 0 & 0 & & $3(75)$ & 0 & 0 & 11 \\
\hline Sinus Bradycardia & 0 & 0 & 0 & $4(100)$ & 0 & 0 & & 0 & 0 & 0 & 4 \\
\hline $\begin{array}{l}\text { Prolong PR } \\
\text { Interval }\end{array}$ & 0 & 0 & $2(11.1)$ & $1(25)$ & 0 & 0 & & 0 & 0 & 0 & 3 \\
\hline Sinus Tachycardia & $7(22.6)$ & 0 & $11(61.1)$ & 0 & 1 & 0 & & 0 & 0 & 0 & 19 \\
\hline Wide QRS & 0 & 0 & $5(27.8)$ & 0 & 0 & 0 & & 0 & 0 & 0 & 5 \\
\hline $\mathrm{T}$ inversion & 0 & 0 & 0 & 0 & 0 & 1 & & 0 & 0 & 0 & 1 \\
\hline ST-T depression & 0 & 0 & 0 & 0 & 0 & 1 & & 0 & 0 & 0 & 1 \\
\hline Tall $\mathrm{R}$ in avR & 0 & 0 & $2(11.1)$ & 0 & 0 & 0 & & 0 & 0 & 0 & 2 \\
\hline Normal ECG & $21(67.7)$ & 2 & $1(5.5)$ & 0 & 0 & 1 & & $1(25)$ & 2 & 0 & 28 \\
\hline Total & $31(100)$ & 2 & 32 & 5 & 1 & 3 & & 4 & 2 & 1 & 81 \\
\hline
\end{tabular}

$\mathrm{n}=$ number of patients

Note: some drug groups induced multiple type of ECG changes in a single patient

Out of 66 poisoning cases 7 patients died. 6 patients of TCA poisoning revealed Prolong QT; 2 of them died. 5 cases $(27.8 \%)$ of TCA poisoning cases developed Wide QRS, of them 2 patients died. Out of $4 \mathrm{~K}$ efflux blocker overdose 3 patients developed Prolong QT and 2 of them died.

\begin{tabular}{cccc} 
Table V: ECG changes associated with mortality & \\
\hline ECG Changes & No of Cases & $\begin{array}{c}\text { Mortality due } \\
\text { to TCA }(\mathbf{n}=\mathbf{1 8})\end{array}$ & $\begin{array}{c}\text { Mortality due } \\
\text { to K efflux } \\
\text { blocker }(\mathbf{n}=\mathbf{4})\end{array}$ \\
\hline Prolong QT & 11 & 2 & 2 \\
Wide QRS & 5 & 2 & \\
Tall R in aVR & 2 & 1 & \\
\hline
\end{tabular}

\section{Discussion}

Poisoning is a global problem and is a medico toxic emergency associated with high mortality rate. Though incidence of acute poisoning in
Bangladesh is gradually increasing, the exact scenario of poisoning is still unknown due to lack of organized poisoning database or national poisoning information center. ${ }^{11}$ A 3-year study conducted in western Bangladesh at Rajshahi Medical College Hospital revealed that $4.13 \%$ of admitted patients were treated due to poisoning. ${ }^{12}$

In this study $86.3 \%$ patients were less than 30 years of age group. Similarly, Dash et alshowed that in India most poisoned patients $(92 \%)$ were less than 40 years of age. ${ }^{13}$ Females $(68.2 \%)$ were predominant in this study. Majority (38 patients, $57.6 \%$ ) of the study population were married. Sobhani AR et al found in their study that $51.4 \%$ were female and $48.6 \%$ were male which is similar to this study. ${ }^{14}$ Female predominance in this study might be due to familial disharmony, poverty, 
failure in the examination, sexual abuse, chronic illness as well as depression leading to selfpoisoning with common drugs.

Sarkar D et al. showed among pharmaceutical agents, benzodiazepines (40\%), antipsychotics (23\%), tricyclic antidepressants (21\%), paracetamol $(8 \%)$ and cetirizine $(4 \%)$ were the most common drugs taken by patients. The most commonly used benzodiazepines ( $42 \%$ of drugs) included diazepam, bromazepam, midazolam and clonazepam. ${ }^{15}$ In our study also benzodiazepines (sedatives) were the most common group (47.0\%) of drugs. This is because in Bangladesh, most medications are easily available in markets and can be sold without prescriptions. In a similar study by Nag et al. revealed that in Bangladesh, diazepam, a benzodiazepine, was the second most common cause of poisoning after OPC; which is consistent with our study. ${ }^{12}$

Majority patients (21 patients, 67.7\%) with benzodiazepine poisoning were found to have normal ECG. Other cases of benzodiazepine overdose revealed sinus tachycardia in $7(22.6 \%)$, prolong QT Interval in 2(6.45\%) and atrial fibrillation in $1(3.23 \%)$ patients. Eizadi-Mood N et al. showed $96.67 \%$ of poisoned patients with benzodiazepines had normal ECG, 5.25\% had sinus tachycardia and $1.87 \%$ had sinus bradycardia. ${ }^{16}$ These findings are similar to our findings other study Worthley Lig et al. showed that in up to $30 \%$ of cases with severe poisoning of benzodiazepines (particularly with respiratory acidosis) there are supraventricular and ventricular arrhythmias. In this study $1(3.23 \%)$ patient with sedative poisoning was found to develop atrial fibrillation, which is a supraventricular arrhythmia.

In patients with TCA (Inhibitors of fast $\mathrm{Na}+$ channel) poisoning, variety of ECG findings were observed including sinus tachycardia in $11(61.1 \%)$, prolong QT interval in $6(33.3 \%)$, wide QRS in $5(27.8 \%)$, atrial tachycardia in 5 (27.8\%), prolong PR interval in $2(11.1 \%)$ and tall $\mathrm{R}$ in aVR in $2(11.1 \%)$ cases. A prospective study by Boehnert et al. in TCA poisoned patients demonstrated that a QRS duration less than 100 $\mathrm{ms}$ was an indicator of good prognosis, while those with a QRS duration over $100 \mathrm{~ms}$ presented with seizures in one third of cases. ${ }^{18}$ Wide QRS
( $>100 \mathrm{~ms}$ ) was found in $27 \%$ of TCA poisoning patients in their study while we found similar changes in $27.8 \%$ cases of TCA poisoning (Table IV). In the same study by Boehnert et al. a QRS complex over $160 \mathrm{~ms}$ was associated with ventricular dysrhythmias. $^{18}$

Harrigan RAet al. showed that ECG changes due to TCA toxicity are attributable primarily to the $\mathrm{Na}^{+}$channel blockade besides sinus tachycardia which is due to anticholinergic effects. The majority of patients with significant risk for developing cardiac or neurological toxicity will have a QRS > $100 \mathrm{~ms}^{19}$

In this study $33.3 \%$ of TCA poisoning patients had prolong QT interval (Figure 2). This finding is consistent with other case reports of TCA poisoning by Vieweg at al. Even among the patients who take therapeutic doses of the tricyclic antidepressants, $20 \%$ may display mild ECG changes including prolongation of QT interval. ${ }^{20,21}$ Other ECG changes associated with TCA overdose are sinus tachycardia, prolonged PR and QRS, ventricular arrhythmias, Brugada sign and ST segment elevation resembling ischaemic heart disease. $^{22}$

$\mathrm{K}+$ efflux channel blocker overdose showed prolong QT Interval in 75\% (3 out of 4) cases (Table IV). Chlorpromazine, chlorpheniramine \& quetiapine, which are $\mathrm{K}+$ efflux channel blockers, were found to display prolong QT Interval in overdoses. Yates $\mathrm{C}$ et al. showed that amitriptyline, nortriptyline, chlorpromazine and quetiapine caused prolong QT Interval which is consistent with the findings of the study. ${ }^{23}$ A case series by Balitet al of 45 quetiapine overdoses revealed Prolong QT in $30 \%$ cases. $^{24}$

Prolong QT intervals are most commonly associated with TCA like amitriptyline and other antidepressants like doxepin, imipramine, and clomipramine poisoning. ${ }^{25}$ In this study also TCA was the most common group that produced prolong QT Interval (6 out of 11 cases of prolong QT Interval) (table IV). Prolong QT Interval was found with higher doses $(750 \mathrm{mg}$ to $1000 \mathrm{mg}$, 3040 tablets) of amitriptyline poisoning. Poisoning with less than 30 tablets ( $<750 \mathrm{mg}$ ) of TCA mostly revealed Sinus Tachycardia (figure 2 ). 
Love JN et al. showed out of 167 patients, 13 were determined to have symptomatic exposures. ${ }^{26}$ First-degree heart block ( $>200 \mathrm{~ms})$ was the most common ECG finding (10/12). But we found all patients with beta blocker overdose revealed sinus bradycardia; while only $1(25 \%)$ patient with 10 tablets of atenolol induced both sinus bradycardia and prolong PR interval; this difference might be due to the lower dose of beta blockers ingested by this study patients.

Overdoses of Paracetamol (500 mg tablets) were observed to display normal ECG, ST-T depression and $\mathrm{T}$ inversion. Similarly Weston $\mathrm{MJ}$ et al. showed that ECG abnormalities, especially of the ST segment or $\mathrm{T}$ wave and Dysrhythmias are frequent findings in paracetamol poisoning. ${ }^{27}$

In this study, one patient with 6 tablets of $0.25 \mathrm{mg}$ digoxin (Na-K ATPase blocker) poisoning was found to have atrial tachycardia. Christopher P et al. showed paroxysmal atrial tachycardia with variable block or accelerated junctional rhythm is highly suggestive of digitalis toxicity. ${ }^{28}$

Yap $G$ et al. showed in his study that antiarrhythmic drugs, non-sedating antihistamines, macrolide antibiotics, antifungals, antimalarials, tricyclic antidepressants, neuroleptics, and prokinetics have all been implicated in causing QT prolongation and/or torsades de pointes. In this study TCA (amitriptylines and nortriptylines), benzodiazepines, chlorpromazine \& anti histamines were found to develop prolong QT Interval which is similar to the study findings by Yap G et al. ${ }^{25}$

This study showed if the quantity of drug consumed by the patients was higher, then specific abnormality occurred ECG. Prolong QT Interval and sinus tachycardia were found in patients who took 20-25 tablets of bromazepam and diazepam (Figure 1). If the quantity of TCA taken were higher (30-40 tablets/750 mg to1000 mg), then ECG findings were abnormal like atrial tachycardia, wide QRS, prolong QT Interval \& prolong PR Interval (Figure 2). Lower doses of TCA $(<30$ tablets/ $<750 \mathrm{mg}$ ) revealed mostly sinus tachycardia.

In adults, the toxic dose of TCAs varies according to the pharmacological characteristics of each one. In case of amitriptyline, low dose of $50 \mathrm{mg}$ may be associated with moderate toxicity and severe toxicity occurs above $300 \mathrm{mg}$, ranging from 150 mg to10 g. ${ }^{29}$ In this study 5 out of 18 patients of TCA overdose (>750 mg of amitriptyline) ultimately died.2 of them had wide QRS, 2 had prolong QT \& 1 had tall R in aVR.27.8\% of TCA poisoning cases revealed wide QRS. Prolong QT was found in $33.3 \%$ of TCA poisoning and $75 \%$ cases of $\mathrm{K}$ efflux blockers overdose. $50 \%$ (cases) of patients with $\mathrm{K}$ efflux blockers overdose died whose ECG showed prolong QT (table V).

In this study 5-10 tablets $(250 \mathrm{mg}$ to $500 \mathrm{mg}$ ) of beta blockers overdose showed sinus bradycardia in $4(100.0 \%)$ cases and prolong PR Interval in $1(25.0 \%)$ case. Anti-histamine produced normal ECG findings even with 20 tablets. Ten tablets of $\mathrm{K}+$ efflux channel blocker (Loratadine) overdose displayed normal ECG, while 20 tablets (chlorpromazine, chlorpheniramine and quetiapine) were found to induce prolong QT Interval.

Though poisoning is a very common cause of hospital admission and mortality, no study demonstrated ECG changes in relation to drug poisoning in the context of Bangladesh. This study reflects that dangerous ECG changes like prolong QT, wide QRS and tall $\mathrm{R}$ in aVR may occur in higher doses of some specific group of drugs. Besides benzodiazepines, TCA is a very common drug group, which is frequently taken by the patients and associated with serious ECG changes. Mortality mostly occurred in patients whose ECG revealed prolong $\mathrm{QT}$, wide $\mathrm{QRS}$ and tall $\mathrm{R}$ in aVR.

\section{Conclusion}

The study showed that amitriptyline and sedatives poisoning were common among young females. These drugs have their own impact and produce different types of ECG changes.ECG is a readily available tool even in remote centres. Attention to specific ECG changes of the poisoning patients can give a clue to the possible underlying drugsand also can guide immediate care. Certain ECG abnormalities also predict the prognosis of the patients. Large scale study with multi stakeholders should be undertaken which will include the effect of elapsed time between the drug ingestion and the ECG changes. 


\section{Acknowledgment}

We gratefully acknowledge the sincere cooperation of the patients as well as the contribution of all doctors, staff of the Department of Medicine, Dhaka Medical College Hospital.

\section{Conflict of interest: None}

\section{References}

1. Albertson TE, Dawson A, de Latorre F. TOX-ACLS: toxicologic-oriented advanced cardiac life support. Annals of Emergency Medicine. 2001;37:S78-90 DOI: doi.org/10.1067/mem.2001.114174

2. Bardin PG, Van Eeden SF, Moolman JA, Foden AP, Joubert JR.Organophosphate and Carbamate poisoning. Archives of Internal Medicine. 1994; 154:1433-41.

DOI: doi.org/10.1001/archinte.154.13.1433

3. Hossain AKMM, Hannan MA, Janan FAJ. Clinical pattern and outcome poisoning- A study in medical indoor of a teaching hospital. Bangladesh Journal of Medicine. 1999;10:27-29.

4. Siwach SB.Organophosporus poisoning-newer challenges. API-medicine update. 1998;177:766-68.

5. Lionte C, Bologa C, Sorodoc L. Toxic and DrugInduced Changes of the Electrocardiogram, Clinical Applications. 2012;371-97. DOI: doi.org/10.5772/22891

6. Liebelt EL, Francis PD, Woolf AD. ECG Lead aVR versus QRS interval in predicting seizures and arrhythmias in acute tricyclic antidepressant toxicity. Annals of Emergency Medicine. 1995;26:195-201. DOI: doi.org/10.1016/S0196-0644(95)70151-6

7. Wolfe TR, Caravati EM, Rollins DE. Terminal 40-ms frontal plane QRS axis as a marker for tricyclic antidepressant overdose. Annals of Emergency Medicine. 1989;18:348-51

DOI: doi.org/10.1016/S0196-0644(89)80566-9

8. Sides GD. QTinterval prolongation as a biomarker for torsades de pointes and sudden death in drug development. Disease Markers. 2002; 18:57-62. DOI: doi.org/10.1155/2002/482953

9. LitonjuaRM. Digoxin: The Monarch of Cardiac Toxicities. Journal of Pharmacy Practice. 2005; 18:157-68.

DOI: doi.org/10.1177/0897190005276751

10. Bazett HC. An analysis of the time-relationship of electro-cardiograms.Heart. 1920; 7:353-70.

11. Bangladesh Ministry of Health and Family Welfare. Health Bulletin 2010. Dhaka: Directorate General of Health Services, Management Information Services. 2010.
12. Nag MN, Jahangir KM, Latif MA, Farid N, Haque A, Khan RK. A Study on Poisoning Cases: Our Experience in Rangpur Medical College Hospital. Northern Medical Journal. 1998; 7:1-4.

13. Das RK. Epidemiology of Insecticide Poisoning at A.I.I.M.S Emergency Services and Role of Its Detection by Gas Liquid Chromatography in Diagnosis. Medico-Legal Update. 2007; 7:49-60.

14. Sobhani AR, Shojaii-Tehrani H, Nikpour E, NorooziRed N. Drug and Chemical poisoning in Northern Iran. Archives of Iranian Medicine. 2000; 3:1-4

15. Sarkar D, Shaheduzzaman M, Hossain MI, Ahmed M, Mohammad N, Basher A. Spectrum of Acute Pharmaceutical and Chemical Poisoning in Northern Bangladesh.Asia pacific journal of medical toxicology. 2013; 2:1.

16. Eizadi-Mood N, Montazeri K, Dastjerdi MD. Evaluation of cardiovascular manifestations in Benzodiazepine poisoning, Iranian Journal of Toxicology, 2011; 4:373-76.

17. Hirsch IA, Zauder HL. Chloral hydrate: a potential cause of arrhythmias. Anesthesia \& Analgesia. 1986; 65:691-92.

DOI: doi.org/10.1213/00000539 - 198606000-00023

18. Boehnert MT, Lovejoy FH Jr. Value of the QRS duration versus the serum drug level in predicting seizures and ventricular arrhythmias after an acute overdose of tricyclic antidepressants. New England Journal Medicine. 1985; 313:174-79. DOI: doi.org/10.1056/NEJM198508223130804

19. Harrigan RA, Brady WJ. ECG abnormalities in tricyclic antidepressant ingestion. American Journal Emergency Medicine. 1999; 17:387-93. DOI: doi.org/10.1016/S0735-6757(99)90094-3

20. Vieweg WV, Wood MA. Tricyclic antidepressants, QT intgerval prolongation, and Torsades de pointes. Psychosomatics. 2004; 45:371-77.

DOI: doi.org/10.1176/appi.psy.45.5.371

21. Groleau G, Jotte R, Barish R. The electrocardiographic manifestations of cyclic antidepressant therapy and overdose: a review. Journal of Emergency Medicine. 1990; 8:597-605. DOI: doi.org/10.1016/0736-4679(90)90457-7

22. Palaniswany C, Selvaraj DR, Chugh T, Singh T, Khalique $\mathrm{O}$, Tsai $\mathrm{F}$, et al. Brugada electrocardiographic pattern induced by amitriptyline overdose. American Journal of Therapeutics. 2010;17:529-32

DOI: doi.org/10.1097/MJT.0b013e3181afbf73

23. Yates C,Manini AF. Utility of the Electrocardiogram in Drug Overdose and Poisoning: Theoretical Considerations and Clinical Implications.Current Cardiology Reviews, 2012;8:137-51 DOI: doi.org/10.2174/157340312801784961 
24. Balit CR, Isbister GK, Hackett PL, Whyte IM. Quetiapine poisoning: a case series. Annals of Emergency Medicine. 2003;42:751-58.

DOI: doi.org/10.1016/S0196-0644(03)00600-0

25. Yap G, Camm AJ. Department of Cardiological Sciences, St Georg's Hospital Medical School, London. UK Heart. 2003; 89:1363-72.

DOI: doi.org/10.1136/heart.89.11.1363

26. Love JN. Enlow B, Howell JM, Klein-Schwartz W, Litovitz TL. Electrocardiographic changes associated with beta-blocker toxicity. Annals of Emergency Medicine. 2002; 40:6036-10.

DOI: doi.org/10.1067/mem.2002.129829

27. Weston MJ, Talbot IC, Howorth PJN, Mant AK,
Capildeo R, Williams R. Frequency of arrhythmias and other cardiac abnormalities in fulminant hepatic failure. British Heart Journal. 1976;38:1179-88.

DOI: doi.org/10.1136/hrt.38.11.1179

28. Lien WC, Huang $\mathrm{CH}$, Chen WJ. Bidirectional ventricular tachycardia resulting from digoxin and amiodarone treatment of rapid atrial fibrillation. American Journal of Emergency Medicine. 2004; 22:235-36.

DOI: doi.org/10.1016/j.ajem.2004.02.034

29. Hulten BA, Heath A, Knudsen K, Nyberg G, Starmark JE, Martens-son E. Severe amitriptyline overdose: relationship between toxicokinetics and toxicodynamics. Journal of Clinical Toxicology. 1992; 30:171-79. 